\title{
On the approximation of positive definite functions by like functions of smaller support
}

\author{
Panagiotis G. Mavroudis
}

Received: 8 April 2013 / Revised: 15 May 2013 / Accepted: 22 May 2013 / Published online: 3 July 2013 (C) The Author(s) 2013. This article is published with open access at SpringerLink.com

\begin{abstract}
Let $\Omega$ be an open 0 -symmetric subset of $\mathbb{R}^{d}$ which contains 0 and $f$ a continuous positive definite function vanishing off $\Omega$, i.e., with supp $f \subseteq \bar{\Omega}$. We study the following problem. Approximate $f$ by a continuous positive definite function $F$ with supp $F \subseteq \Omega$, that is by a function which is vanishing in a neighborhood of the boundary. This is very easy to do when $\Omega$ is strictly star-shaped with respect to 0 . We give a proof that this is indeed possible when $\Omega \subseteq \mathbb{R}$ is finite union of intervals and we extend the result to $\mathbb{R}^{d}$ for functions with radial symmetry and $\Omega$ is a finite union of annular regions.
\end{abstract}

Mathematics Subject Classification (2010) $42 B 99$

\section{Introduction}

1.1 The Turán-Stechkin extremal problem

The following extremal problem has been proposed by Turán and Stechkin [12]:

Given an open set $\Omega \subseteq \mathbb{R}^{d}$, symmetric about 0 , and a continuous, positive definite, integrable function $f$, with supp $f \subseteq \Omega$ and with $f(0)=1$, how large can $\int f$ be?

Communicated by S. K. Jain.

\section{P. G. Mavroudis}

Department of Mathematics, University of Crete, Knossos Ave., 71409 Heraklion, Greece e-mail: mavroud@math.uoc.gr 
Many cases studied so far concern $\Omega$ being a convex subset of $\mathbb{R}^{d}[2-4,8]$. The conjectured extremum in this case, arising when we take

$$
f=\frac{2^{d}}{|\Omega|} \chi_{\frac{1}{2} \Omega} * \chi_{\frac{1}{2} \Omega},
$$

is $\frac{|\Omega|}{2^{d}}$ (here $\chi_{E}$ denotes the indicator function of a set $E$ ). The problem also makes sense in other abelian groups $[9,10]$. For instance, one can take $\Omega$ to be an interval in the torus $\mathbb{T}=\mathbb{R} / \mathbb{Z}[5,12,6,7,1]$. This extremal problem remains essentially open in dimension $d \geq 2$ and it is only in special cases where we know the answer, even when examining only convex domains $\Omega$.

\subsection{Approximation by positive definite functions of smaller support}

When one considers, as in the Turán-Stechkin problem above, positive definite functions $f$ with support in $\Omega$, an open set in $\mathbb{R}^{d}$, symmetric with respect to 0 , the question arises whether we allow the support of $f$ to touch the boundary or not. In other words do we demand that the closed set supp $f$ is contained in the open set $\Omega$ or in the closed set $\bar{\Omega}$ ?

In many cases in analysis such a question would be meaningless as one can usually approximate functions with support on $\bar{\Omega}$ by functions supported in $\Omega$ proper. However, when one requires that the approximating functions are also positive definite this property is by no means evident. It is exactly this question that we try to answer in this paper, for some special classes of domains.

Main question: For a given $\Omega \subseteq \mathbb{R}^{d}$ (containing 0 , open and symmetric with respect to 0 ) and a continuous positive definite function $f: \mathbb{R}^{d} \rightarrow \mathbb{C}$ which vanishes off $\Omega$, when can we approximate $f$ uniformly by continuous positive definite functions whose support is contained in $\Omega$ ?

Apart from the Turán-Stechkin problem mentioned above this question could, for instance, have implications for the approximation of band-limited probability distributions (in the general sense of restricting the Fourier support).

The answer turns out to be very simple in the case when $\Omega$ can be "shrunk" into itself.

Call an open set $\Omega \subseteq \mathbb{R}^{d}$ strictly star-shaped (with respect to the origin) if $\rho \bar{\Omega} \subseteq \Omega$ for any $0 \leq \rho<1$.

Theorem 1 Let $\Omega \subseteq \mathbb{R}^{d}$ be an open, bounded, strictly star-shaped and symmetric set, and assume that $\epsilon>0$ and $f$ is a continuous, positive definite function which vanishes outside $\Omega$ (i.e. supp $f \subseteq \bar{\Omega}$ ). Then there is a continuous positive definite function $F$, with supp $F \subseteq \Omega$, such that

$$
\|f-F\|_{\infty} \leq \epsilon
$$


Proof Take $\epsilon>0$ and $F(x)=f\left((1-\epsilon)^{-1} x\right)$. This is positive definite, has support in $\Omega$ by the strict star-shaped property of $\Omega$ and tends to $f$ in the uniform norm for $\epsilon \rightarrow 0$.

We do not know if this is true if we remove the geometric assumption that $\Omega$ is strictly star-shaped.

\subsection{Notation:}

- The letter $C$ denotes a positive constant which need not be the same wherever it appears.

- $C^{k}$ denotes the space of functions on $\mathbb{R}^{d}$ with continuous derivatives up to order k. $C^{\infty}=\bigcup_{k \geq 0} C^{k}$ (smooth functions) and $C_{c}^{\infty}$ denotes smooth functions with compact support.

- For $f \in L^{1}\left(\mathbb{R}^{d}\right)$ the Fourier Transform of $f$ is the function $\widehat{f}(\xi)=$ $\int e^{-2 \pi i \xi \cdot x} f(x) d x$.

- If $x \in \mathbb{R}^{d}$ we denote by $\delta_{x}$ the unit measure at $x$ (Dirac mass).

- For $E \subseteq \mathbb{R}^{d}$ we denote by $E^{c}$ its complement.

- In the proofs that follow $\phi: \mathbb{R}^{d} \rightarrow \mathbb{R}$ always denotes a continuous positive definite function supported in the unit ball of $\mathbb{R}^{d}$ with integral 1 and, for $\epsilon>0$, we write $\phi_{\epsilon}(x)=\epsilon^{-d} \phi(x / \epsilon)$, for the dilate of the function supported in the central ball of radius $\epsilon$ and again with integral 1 . The dimension $d$ will be clear from the context.

\subsection{The problem in dimension 1}

The problem is just as valid in dimension 1, where it is not very hard to deal with. The reason is that in dimension 1 the boundary is very small.

Theorem 2 Suppose $0 \in \Omega \subseteq \mathbb{R}$ is a finite union of open intervals, $\Omega=-\Omega$ and $f$ is a continuous, positive definite function, and with supp $f \subseteq \bar{\Omega}$ and $\delta>0$. Then there is a continuous positive definite function $F$, supported in $\Omega$ (that is, away from the boundary) such that $\|f-F\|_{\infty}<\delta$.

Proof Let $A$, a finite set, be the set of endpoints of the intervals in $\Omega$, and let $\epsilon>0$ be small. Write $h(\epsilon)$ for the supremum of $|f|$ in an $\epsilon$-neighborhood of $A$. Notice that $h(\epsilon) \rightarrow 0$ as $\epsilon \rightarrow 0$ by the continuity of $f$ and the fact that $\Omega$ is bounded. Write also $N=\# A$ and observe that the measure

$$
\mu=g+(\epsilon h(\epsilon) N) \delta_{0}
$$

is positive definite, where $g$ is the function $f$ set to 0 in an $\epsilon$-neighborhood of $A$ and $\delta_{0}$ the Dirac mass at the origin.

This measure is supported $\epsilon$-away from $A$. 
Define the positive definite function $F=\mu * \phi_{\epsilon / 2}$ which is supported in $\Omega$ (in fact $(\epsilon / 2)$-away from the boundary). Then

$$
\begin{aligned}
F(x) & =\left(g * \phi_{\epsilon / 2}\right)(x)+(\epsilon h(\epsilon) N) \phi_{\epsilon / 2}(x) \\
& =\left(g * \phi_{\epsilon / 2}\right)(x)+(2 h(\epsilon) N) \phi(2 x / \epsilon) .
\end{aligned}
$$

Observe that the second term tends uniformly to 0 as $\epsilon \rightarrow 0$. Finally if $x$ is farther than $3 \epsilon / 2$ from the boundary then $\left(g * \phi_{\epsilon / 2}\right)(x)=\left(f * \phi_{\epsilon / 2}\right)(x)$ so, in that case,

$$
f(x)-F(x)=f(x)-\left(f * \phi_{\epsilon / 2}\right)(x)+o(1)
$$

which tends to 0 with $\epsilon$. Since both $F$ and $f$ are $o(1)$ for $x$ near the boundary, it follows that $F$ can be made arbitrarily close to $f$ in the uniform norm.

Corollary 1 Let $E=\bigcup_{n=0}^{\infty}\left(a_{n}, b_{n}\right),\left(a_{0}, b_{0}\right)$ being a 0 -symmetric interval and $a_{0}<$ $b_{0}<a_{1}<\ldots$ Set $\Omega=E \cup-E$. Suppose that $\epsilon>0$ and that $f$ is a continuous, positive definite and integrable function supported in $\bar{\Omega}$. Then there exists a compactly supported continuous positive definite function $F$ such that

$$
\|f-F\|_{\infty} \leq \epsilon
$$

Proof We have $\hat{f} \in L^{1}(\mathbb{R})$ ( $f$ being positive definite, continuous and $L^{1}$ ). Therefore, by the Riemann-Lebesgue lemma, we have $f(x) \rightarrow 0$ as $|x| \rightarrow \infty$. For any $\epsilon>0$ the function $(1-|\epsilon x|)_{+}$is compactly supported, positive definite and approximates 1 uniformly on compact sets as $\epsilon \rightarrow 0$. Hence the function $f_{\epsilon}(x)=(1-|\epsilon x|)_{+} f(x)$ is positive definite, approximates $f$ uniformly on $\mathbb{R}$ and

$$
\operatorname{supp} f_{\epsilon} \subseteq \bar{\Omega} \cap\left[-\frac{1}{\epsilon}, \frac{1}{\epsilon}\right]
$$

Since $f_{\epsilon}$ is supported in a finite union of intervals we can use Theorem 2 to obtain a positive definite function $F$ with support away from the endpoints of these intervals which approximates $f_{\epsilon}$, and hence $f$ itself, uniformly on $\mathbb{R}$.

The method fails in dimension 2 and higher as $\phi_{\epsilon}$, as used in the proof of Theorem 2 , now becomes of the order of $\epsilon^{-2}$ at 0 and the second term in (1) above is not $o$ (1) any longer. Our main result in dimension 2 and higher concerns functions with radial symmetry and is presented in Sect. 2.

\section{The case of radial functions}

Our main result in this paper is the following. 
Theorem 3 Suppose $f: \mathbb{R}^{d} \mapsto \mathbb{R}$ is a radial, continuous and positive definite function with compact support. Let $0<m_{0}<r_{1}<m_{1}<\cdots<r_{N}<m_{N}$. Suppose that supp $f \subseteq \bar{\Omega}$ where

$$
\Omega=\left\{x \in \mathbb{R}^{d}:|x|<m_{0}\right\} \cup \bigcup_{k=1}^{N}\left\{x \in \mathbb{R}^{d}: r_{k}<|x|<m_{k}\right\} .
$$

Then, given $\delta>0$, there is a positive definite, continuous radial function $F$, supported in $\Omega$, such that $\|f-F\|_{\infty}<\delta$.

\subsection{The proof in dimension $d=2$ or 3}

We first give a proof of Theorem 3 for $d=2,3$ and then we give another proof which is valid for all $d \geq 2$. We could have given only the second proof but we prefer to give them both as the first is simpler (but breaks down at $d=4$ ).

The symbol $C$ stands for an absolute constant, not necessarily the same in all its occurences. We write $f(r):=f(x)$ when $|x|=r$. For $\epsilon>0$ we consider the subsets of $\Omega$, near its boundary,

$$
A_{1}=\bigcup_{k=1}^{N}\left\{x \in \mathbb{R}^{d}: r_{k}<|x|<r_{k}+\epsilon\right\}
$$

and

$$
A_{2}=\bigcup_{k=0}^{N}\left\{x \in \mathbb{R}^{d}: m_{k}-\epsilon<|x|<m_{k}\right\} .
$$

We take $\epsilon>0$ small enough to have $A_{1} \cap A_{2}=\emptyset$ and set $U=A_{1} \bigcup A_{2}$. Let $g$ be the function $f$ set to 0 on $U$ and $h(\epsilon)=\sup _{x \in U}|f(x)|$, as in the proof of Theorem 2 . For sufficiently small $\epsilon>0$ we can find $\delta_{j}, j=1,2$, such that

$$
\delta_{2}<1<\delta_{1}, \quad \delta_{j} A_{j} \subseteq \Omega, \quad \operatorname{dist}\left(\delta_{j} A_{j}, \partial \Omega\right) \geq \epsilon, \text { and }\left|1-\delta_{j}\right|=O(\epsilon)
$$

We consider the function

$$
G(x)=g(x)+\frac{1}{\delta_{1}^{d}}\left(f \chi_{A_{1}}\right)\left(x / \delta_{1}\right)+\frac{1}{\delta_{2}^{d}}\left(f \chi_{A_{2}}\right)\left(x / \delta_{2}\right),
$$

which is supported in $\Omega$ and at distance $\geq \epsilon$ from $\partial \Omega$. On the Fourier Transform side we have 


$$
\begin{aligned}
& \widehat{G}(\xi)=\widehat{g}(\xi)+\widehat{f \chi_{A_{1}}}\left(\delta_{1} \xi\right)+\widehat{f \chi_{A_{2}}}\left(\delta_{2} \xi\right) \\
& =\widehat{f}(\xi)-\int_{U} f(x) e^{-2 \pi i x \xi} d x+\widehat{f \chi_{A_{1}}}\left(\delta_{1} \xi\right)+\widehat{f \chi_{A_{2}}}\left(\delta_{2} \xi\right) \\
& \geq-\int_{A_{1}} f(x) e^{-2 \pi i x \xi} d x-\int_{A_{2}} f(x) e^{-2 \pi i x \xi} d x+\int_{A_{1}} f(x) e^{-2 \pi i \delta_{1} x \xi} d x \\
& \quad+\int_{A_{2}} f(x) e^{-2 \pi \delta_{2} i x \xi} d x .
\end{aligned}
$$

Observe that (writing $t=|x|$ )

$$
\begin{aligned}
I_{1} & =\left|\int_{A_{1}} f(x) e^{-2 \pi i x \xi} d x-\int_{A_{1}} f(x) e^{-2 \pi i x \delta_{1} \xi} d x\right| \\
& =\left|\sum_{k=1}^{N} \int_{r_{k}<|x|<r_{k}+\epsilon} f(x)\left(e^{-2 \pi i x \xi}-e^{-2 \pi i x \delta_{1} \xi}\right) d x\right| \\
& \leq \sum_{k=1}^{N}\left|\int_{r_{k}<t<r_{k}+\epsilon} t^{d-1} f(t)\left(\widehat{\sigma}(t|\xi|)-\widehat{\sigma}\left(\delta_{1} t|\xi|\right)\right) d t\right|
\end{aligned}
$$

where $\widehat{\sigma}(t)=\widehat{\sigma_{d-1}}(\xi)$ is the Fourier Transform of the surface measure on the unit sphere in $\mathbb{R}^{d}$. We have used here the Fourier integral for a radial function $g$ in the form that arises from polar integration

$$
\widehat{g}(\xi)=\int g(x) e^{-2 \pi i \xi \cdot x} d x=\int_{0}^{\infty} g(t) t^{d-1} \widehat{\sigma}(|\xi| t) d t,
$$

with the obvious identification $g(|x|)=g(x)$ for a radial function $g$.

Consider the function $K(s)=\widehat{\sigma}(s t|\xi|)$. By the mean value theorem we have for some $s^{*}$ between 1 and $\delta_{1}$

$$
\left|K(1)-K\left(\delta_{1}\right)\right|=\left(\delta_{1}-1\right)\left|K^{\prime}\left(s^{*}\right)\right|=\left(\delta_{1}-1\right) t|\xi| \widehat{\sigma}^{\prime}\left(s^{*} t|\xi|\right) .
$$

Since $\widehat{\sigma}(r), \widehat{\sigma}^{\prime}(r)=O\left((1+r)^{-(d-1) / 2}\right)$ as $r \rightarrow \infty[11$, Theorem 1.2.1] and $\left|1-\delta_{1}\right|=O(\epsilon)$ it follows that

$$
\left|\widehat{\sigma}(t|\xi|)-\widehat{\sigma}\left(\delta_{1} t|\xi|\right)\right| \leq C \epsilon|\xi|(1+|\xi|)^{-(d-1) / 2}
$$

for all $t \in \cup_{k=1}^{N}\left(r_{k}, r_{k}+\epsilon\right)$. We also have, by the asymptotics for $\widehat{\sigma}$, that

$$
\left|\widehat{\sigma}(t \xi)-\widehat{\sigma}\left(\delta_{1} t \xi\right)\right| \leq C(1+|\xi|)^{-(d-1) / 2} .
$$


It follows that

$$
I_{1} \leq C \min \left\{\epsilon h(\epsilon)(1+|\xi|)^{-(d-1) / 2}, \epsilon h(\epsilon) \epsilon|\xi|(1+|\xi|)^{-(d-1) / 2}\right\}
$$

When $d=2$ or $d=3$ and $|\xi| \leq 1 / \epsilon$ the second term in the minimum above is smaller and is an increasing function of $|\xi|$ in that range (it is not increasing when $d \geq 4$ ). The first function in the minimum is decreasing in $|\xi|$, so in dimensions 2 and 3 we have

$$
I_{1} \leq C \epsilon^{(d+1) / 2} h(\epsilon), \quad(d=2,3)
$$

In the same way we estimate the difference of integrals over $A_{2}$. Therefore we have proved for $d \leq 3$ that

$$
\widehat{G}(\xi) \geq-C \epsilon^{(d+1) / 2} h(\epsilon)
$$

or that the measure

$$
\mu=G+\left(C \epsilon^{(d+1) / 2} h(\epsilon)\right) \delta_{0}
$$

is positive definite. The support of this measure is in $\Omega$ and $\epsilon$-away from the boundary. Take $\phi$ supported in $D(0,1 / 5)$, the open ball of radius $1 / 5$ centered at 0 , positive definite, to be radial, continuous and with integral 1 . We will show that the positive definite function

$$
\phi_{\epsilon} * \mu=\phi_{\epsilon} * G+\frac{h(\epsilon)}{\epsilon^{(d-1) / 2}} \phi(x / \epsilon)
$$

converges uniformly to $f$ on $\mathbb{R}^{d}$ as $\epsilon \rightarrow 0$. First, observe that $\phi_{\epsilon} * G(x)=\phi_{\epsilon} * f(x)$ if $\operatorname{dist}(x, \partial \Omega)>2 \epsilon$, hence $\phi_{\epsilon} * G \rightarrow f$ uniformly in $\mathbb{R}^{d}$, as both $f$ and $G$ are $o(1)$ at distance $2 \epsilon$ from the boundary (as $\epsilon \rightarrow 0$ ).

Therefore it is enough to prove that

$$
h(\epsilon)=o\left(\epsilon^{(d-1) / 2}\right), \text { as } \epsilon \rightarrow 0,(d \geq 1),
$$

so that the second term in (3) tends uniformly to 0 on $\mathbb{R}^{d}$. We present the proof below following the approach of [13], for all dimensions $d \geq 1$.

Write $Y(r)=\widehat{f}(\xi)$ when $|\xi|=r$ and observe that, since $\widehat{f} \in L^{1}\left(\mathbb{R}^{d}\right)$ (as $f$ is positive definite and $f(0)=1$ ), it follows using polar integration that

$$
\int_{0}^{\infty} r^{d-1}|Y(r)| d r<\infty .
$$


Rewriting the Fourier inversion formula on $\mathbb{R}^{d}$ in polar coordinates, where we abuse notation writing $f(t)$ for the value of the radial $f$ at any $x$ with $|x|=t$, we get

$$
f(t)=\int_{0}^{\infty} r^{d-1} Y(r) \widehat{\sigma}(r t) d r
$$

Since (see [11, Theorem 1.2.1]) we have

$$
\left|\left(\frac{d}{d t}\right)^{a} \hat{\sigma}(t)\right| \leq \frac{C_{a}}{(1+|t|)^{(d-1) / 2}}, \quad \text { for any } a \in \mathbb{N},
$$

it follows from $(5)$ that we can "differentiate $(d-1) / 2$ times" under the integral sign in (6). Define

$$
\ell=\left|\frac{d-1}{2}\right|
$$

It then follows that $f \in C^{\ell}(0, \infty)$ and we can write

$$
f^{(\ell)}(t)=\int_{0}^{\infty} r^{d-1+\ell} Y(r) \widehat{\sigma}^{(\ell)}(r t) d r
$$

In other words, we can differentiate under the integral sign $\ell$ times since the successive derivatives are integrable, due to (7) and (5).

The case $d=2 \ell+1$ being odd. Since $f \in C^{\ell}(0, \infty)$ and at $r=r_{j}$ (resp. $r=m_{j}$ ), $j \geq 1$ (resp. $j \geq 0$ ), all side derivatives on the left (resp. right) are 0 , as the function $f$ is identically 0 there, it follows that the first $\ell$ derivatives of $f(r)$ at $r=r_{j}$ or $r=m_{j}$ are 0 . This proves (4) for the case of odd $d$ (the Taylor polynomial of order $\ell$ is identically 0 at $r_{j}, m_{j}$, and therefore $f$ is $o\left(\epsilon^{\ell}\right)=o\left(\epsilon^{(d-1) / 2}\right)$ at distance $\epsilon$ from $r_{j}$ or $m_{j}$ ).

The case $d=2 \ell+2$ being even. We have $\frac{d-1}{2}=\ell+\frac{1}{2}$. To prove (4) it is enough to show that

$$
\lim _{\delta \rightarrow 0} \frac{1}{\delta^{1 / 2}}\left(f^{(\ell)}(t+\delta)-f^{(\ell)}(t)\right)=0, \quad \text { for all } t>0 .
$$

Using (8) we have that the left-hand side of (9) is equal to

$$
\lim _{\delta \rightarrow 0} \int_{0}^{\infty} r^{\frac{3}{2}(d-1)} Y(r)(r \delta)^{-1 / 2}\left(\widehat{\sigma}^{(\ell)}(r t+r \delta)-\widehat{\sigma}^{(\ell)}(r t)\right) d r
$$


and we can interchange the limit with the integral sign if

$$
(r \delta)^{-1 / 2}\left|\widehat{\sigma}^{(\ell)}(r t+r \delta)-\widehat{\sigma}^{(\ell)}(r t)\right| \leq \frac{C}{(1+r)^{(d-1) / 2}}
$$

for a finite constant $C$ (may depend on $t$ ), using (5) and the dominated convergence theorem. If $|r \delta| \geq 1$ then it follows from (7) that the left hand side of (10) is bounded by $K_{1}(1+r)^{-(\bar{d}-1) / 2}$ for some finite constant $K_{1}>0$ (which may depend on $t$ ). If $|r \delta| \leq 1$ then, using (7) and the mean value theorem we bound the left hand side of (10) by $K_{2}(1+r)^{-(d-1) / 2}$, for some finite constant $K_{2}>0$ (which, again, may depend on $t$ only). This concludes the proof of (10) and (9) follows since the differentiability of $\widehat{\sigma}^{(\ell)}$ implies

$$
\lim _{\delta \rightarrow 0}(r \delta)^{-1 / 2}\left(\widehat{\sigma}^{(\ell)}(r t+r \delta)-\widehat{\sigma}^{(\ell)}(r t)\right)=0, \text { for all } \mid r, t \geq 0
$$

The proof of (4) is now complete and Theorem 3 is proved for $d=2,3$.

\subsection{The proof of Theorem 3 for all $d \geq 2$}

We now give a modification of the previous proof which works for all $d \geq 2$. We will again "erase" the function $f$ near the boundary and will compensate by adding appropriate functions supported properly inside $\Omega$ so that the function $G$ (which plays the same role as in Sect. 2.1) again satisfies (2). From that point on the proof proceeds as in Sect. 2.1.

For $\epsilon>0$ small take a radial function $\eta \in C_{c}^{\infty}$ such that

$$
\begin{gathered}
\eta(x)=1, \quad \text { if } r_{k} \leq|x| \leq r_{k}+\epsilon / 2 \text { or } m_{k}-\epsilon / 2 \leq|x| \leq m_{k} \\
\eta(x)=0, \quad \text { if }|x| \leq m_{0}-\epsilon \text { or } r_{k}+\epsilon \leq|x| \leq m_{k}-\epsilon \text { and } \\
0 \leq \eta(x) \leq 1, \quad \text { everywhere. }
\end{gathered}
$$

It follows that the support of the function $f-\eta f$ is $\epsilon / 2$-away from the boundary. We know that $f \in C^{\ell}(0,+\infty)$ and so is $\eta f$.

Separate the support of $f \eta$ as $A_{1} \bigcup A_{2}$ and take $\delta_{1}, \delta_{2}$ as in the previous argument. Define the radial function $\psi_{j}^{k}(r)$, by

$$
\psi_{j}^{k}(r)=\frac{1}{r^{d-1}} \frac{d^{k}}{d r^{k}}\left[r^{d-1+k} f(r) \eta(r)\right] \chi_{A_{j}}(r), \quad(\text { for } k=1,2, \ldots, \ell, \quad j=1,2)
$$


Consider now the function

$$
\begin{aligned}
G & =f-\eta f+\sum_{j=1}^{2}\left(\eta f \chi_{A_{j}}\right)_{\delta_{j}}+\sum_{j=1}^{2} \sum_{k=1}^{\ell} \frac{(-1)^{k}}{k !} \frac{\left(1-\delta_{j}\right)^{k}}{\left(\delta_{j}\right)^{k}}\left(\psi_{j}^{k}\right)_{\delta_{j}} \\
& =f-\sum_{j=1}^{2}\left(\eta f \chi_{A_{j}}-\left(\eta f \chi_{A_{j}}\right)_{\delta_{j}}\right)+\sum_{j=1}^{2} \sum_{k=1}^{\ell} \frac{(-1)^{k}}{k !} \frac{\left(1-\delta_{j}\right)^{k}}{\left(\delta_{j}\right)^{k}}\left(\psi_{j}^{k}\right)_{\delta_{j}},
\end{aligned}
$$

where, as usual, $\phi_{\delta}(x)=\delta^{-d} \phi(x / \delta)$. The function $G$ is supported in $\Omega$ and $\epsilon / 2$-away from the boundary. Observe that

$$
\begin{aligned}
& \widehat{\left(\psi_{1}^{k}\right)_{\delta_{1}}}(\xi)=\sum_{n} \int_{r_{n}}^{r_{n}+\epsilon} r^{d-1} \psi_{1}^{k}(r) \widehat{\sigma}\left(\delta_{1} r|\xi|\right) d r \\
& =\sum_{n} \int_{r_{n}}^{r_{n}+\epsilon} \frac{d^{k}}{d r^{k}}\left(r^{d-1+k} f(r) \eta(r)\right) \widehat{\sigma}\left(\delta_{1} r|\xi|\right) d r \\
& =(-1)^{k} \sum_{n} \int_{r_{n}}^{r_{n}+\epsilon} r^{d-1+k} f(r) \eta(r) \frac{d^{k}}{d r^{k}}\left(\widehat{\sigma}\left(\delta_{1} r|\xi|\right)\right) d r \quad \text { (integrating by parts) } \\
& =(-1)^{k} \sum_{n} \int_{r_{n}}^{r_{n}+\epsilon} r^{d-1+k} f(r) \eta(r) \delta_{1}^{k}|\xi|^{k} \widehat{\sigma}(k)\left(\delta_{1} r|\xi|\right) d r \\
& =\left.(-1)^{k} \sum_{n} \int_{r_{n}}^{r_{n}+\epsilon} r^{d-1} f(r) \eta(r) \delta_{1}^{k} \frac{d^{k}}{d s^{k}} \widehat{\sigma}(s r|\xi|)\right|_{s=\delta_{1}} d r .
\end{aligned}
$$

For the Fourier transform of $\left(\psi_{2}^{k}\right)_{\delta_{2}}$ we work similarly.

Therefore we have

$$
\begin{aligned}
& \left|\widehat{\eta f \chi_{A_{1}}}(\xi)-\widehat{\eta f \chi_{A_{1}}}\left(\delta_{1} \xi\right)-\sum_{k=1}^{\ell} \frac{(-1)^{k}}{k !} \frac{\left(1-\delta_{1}\right)^{k}}{\left(\delta_{1}\right)^{k}} \widehat{\left(\psi_{1}^{k}\right)_{\delta_{1}}}(\xi)\right| \\
& =\mid \sum_{n} \int_{r_{n}}^{r_{n}+\epsilon} r^{d-1} f(r) \eta(r)\left(\widehat{\sigma}(r|\xi|)-\widehat{\sigma}\left(\delta_{1} r|\xi|\right)\right. \\
& \left.\quad-\ldots-\left.\frac{\left(1-\delta_{1}\right)^{\ell}}{\ell !} \frac{d^{\ell}}{d s^{\ell}} \widehat{\sigma}(s r|\xi|)\right|_{s=\delta_{1}}\right) d r \mid,
\end{aligned}
$$


as well as

$$
\begin{aligned}
& \left|\widehat{\eta f \chi_{A_{2}}}(\xi)-\widehat{\eta f \chi_{A_{2}}}\left(\delta_{2} \xi\right)-\sum_{k=1}^{\ell} \frac{(-1)^{k}}{k !} \frac{\left(1-\delta_{2}\right)^{k}}{\left(\delta_{2}\right)^{k}} \widehat{\left(\psi_{2}^{k}\right)_{\delta_{2}}}(\xi)\right| \\
& =\mid \sum_{n} \int_{m_{n}-\epsilon}^{m_{n}} r^{d-1} f(r) \eta(r)\left(\widehat{\sigma}(r|\xi|)-\widehat{\sigma}\left(\delta_{2} r|\xi|\right)\right. \\
& \left.\quad-\ldots-\left.\frac{\left(1-\delta_{2}\right)^{\ell}}{\ell !} \frac{d^{\ell}}{d s^{\ell}} \widehat{\sigma}(s r|\xi|)\right|_{s=\delta_{2}}\right) d r \mid .
\end{aligned}
$$

Consider the function $K(s)=\widehat{\sigma}(s r|\xi|)$. By Taylor's theorem we have for some $s_{j}^{*}$ between 1 and $\delta_{j}$ (for $j=1,2$ again)

$$
\begin{aligned}
& \left|K(1)-K\left(\delta_{j}\right)-\cdots-\frac{\left(1-\delta_{j}\right)^{\ell}}{\ell !} K^{(\ell)}\left(\delta_{j}\right)\right|=\left|\frac{\left(1-\delta_{j}\right)^{\ell+1}}{(\ell+1) !} K^{(\ell+1)}\left(s_{j}^{*}\right)\right| \\
& =\left.\left|r^{\ell+1}\right| \xi\right|^{\ell+1} \frac{\left(1-\delta_{j}\right)^{\ell+1}}{(\ell+1) !} \widehat{\sigma}^{(\ell+1)}\left(s_{j}^{*} r|\xi|\right) \mid .
\end{aligned}
$$

Since $\left|1-\delta_{j}\right|=O(\epsilon)$ and $\left|\left(\frac{d}{d t}\right)^{(\ell+1)} \widehat{\sigma}(t)\right| \leq C(1+|t|)^{-\frac{d-1}{2}}$ it follows that the above quantity (13) is bounded by

$$
C \epsilon^{\ell+1}(1+|\xi|)^{\ell+1-\frac{d-1}{2}} .
$$

Using $\ell+1-\frac{d-1}{2}>0$ we obtain, for $j=1,2$, that

$$
\begin{aligned}
& \mid \widehat{\eta f \chi_{A_{j}}}(\xi)-\widehat{\eta f \chi_{A_{j}}}\left(\delta_{j} \xi\right) \\
& \quad+\sum_{k=1}^{\ell} \frac{(-1)^{k}}{k !} \frac{\left(1-\delta_{j}\right)^{k}}{\left(\delta_{j}\right)^{k}} \widehat{\left(\psi_{j}^{k}\right)_{\delta_{j}}}(\xi) \mid \leq C \epsilon^{\frac{d+1}{2}} h(\epsilon), \text { when }|\xi| \leq \frac{1}{\epsilon}
\end{aligned}
$$

The same inequality holds for $|\xi|>\frac{1}{\epsilon}$ by using (11) or (12).

We have proved that $\widehat{G} \geq-C \epsilon^{\frac{d+1}{2}} h(\epsilon)$. The remainder of the proof is exactly as in the cases $d=2,3$ that we considered earlier in Sect. 2.1, namely we add an appropriate Dirac mass at the origin and then convolve the resulting positive definite measure with a smooth positive definite function of small support.

The proof of Theorem 3 is now complete.

Acknowledgments I would like to thank Prof. M. Kolountzakis for essential help and support in this work.

Open Access This article is distributed under the terms of the Creative Commons Attribution License which permits any use, distribution, and reproduction in any medium, provided the original author(s) and the source are credited. 


\section{References}

1. Andreev, N., Konyagin, S.V., Popov, A.Yu.: Maximum problems for functions with small support. Math. Notes 60(3), 241-247 (1996)

2. Arestov, V.V., Berdysheva, E.E.: Turán's problem for positive definite functions with supports in a hexagon. Proc. Steklov Inst. Math., Suppl. 1, S20-S29 (2001)

3. Arestov, V.V., Berdysheva, E.E.: The Turán problem for a class of polytopes. East J. Approx. 8(3), 381-388 (2002)

4. Gorbachev, D.V.: An extremal problem for periodic functions with supports in the ball. Math. Notes 69(3), 313-319 (2001)

5. Gorbachev, D.V., Manoshina, A.S.: Problem, Turán Extremum, for Periodic Function with Small Support, preprint. arXiv:math.CA/0211291 v1 19 Nov 2002; Tchebyshev Collection. TPSU (Russia) 2, 31-40 (2001)

6. Ivanov, V.I.: On the Turán and Delsarte problems for periodic positive definite functions. Math. Notes 80(6), 875-880 (2006) (translation of Mat. Zametki 80 (2006) 6, 934-939)

7. Ivanov, V.I., Rudomazina, Yu.D.: On the Turán problem for periodic functions with nonnegative Fourier coefficients and small support. Math. Notes 77(6), 870-875 (2005) (translation of Mat. Zametki 77(2005), 6, 941-945)

8. Kolountzakis, M.N., Révész, Sz.Gy.: On a problem of Turán about positive definite functions. Proc. Am. Math. Soc. 131, 3423-3430 (2003)

9. Kolountzakis, M.N., Révész, Sz.Gy.: Turán's extremal problem for positive definite functions on groups. J. Lond. Math. Soc. 74(2), 475-496 (2006)

10. Révész, Sz.Gy: Turán's extremal problem on locally compact abelian groups. Anal. Math. 37(1), 15-50 (2011)

11. Sogge, C.D.: Fourier Integrals in Classical Analysis. Cambridge University Press, Cambridge (1993)

12. Stechkin, S.B.: An extremal problem for trigonometric series with nonnegative coefficients. Acta Math. Acad. Sci. Hung. 23(3-4), 289-291 (1972). Russian

13. Wendland, H.: On the smoothness of positive definite and radial functions. J. Comp. Appl. Math. 101(1-2), 177-188 (1999) 